\title{
Radionuclide Residual Syringe Counts
}

National Cancer Institute

\section{Source}

National Cancer Institute. Radionuclide Residual Syringe Counts. NCI Thesaurus. Code C69293.

The amount of radioactive material remaining in a syringe after administration, measured as counts per minute. 